\title{
Ovine uterine space restriction alters placental transferrin receptor and fetal iron status during late pregnancy
}

\author{
Mary Y. Sun 1,2, Jason M. Habeck', Katie M. Meyer ${ }^{1-3}$, Jill M. Koch², Jayanth Ramadoss², Sharon E. Blohowiak', \\ Ronald R. Magness ${ }^{1-3}$ and Pamela J. Kling'
}

BACKGROUND: Fetal growth restriction is reported to be associated with impaired placental iron transport. Transferrin receptor (TfR) is a major placental iron transporter in humans but has not been studied in sheep. TfR is regulated by both iron and nitric oxide (NO), the molecule produced by endothelial nitric oxide synthase (eNOS). We hypothesized that limited placental development downregulates both placental TfR and eNOS expression, thereby lowering fetal tissue iron.

METHODS: An ovine surgical uterine space restriction (USR) model, combined with multifetal gestation, tested the extremes of uterine and placental adaptation. Blood, tissues, and placentomes from non-space restricted (NSR) singletons were compared with USR fetuses at gestational day (GD) 120 or 130.

RESULTS: When expressed proportionate to fetal weight, liver iron content did not differ, whereas renal iron was higher in USR vs. NSR fetuses. Renal TfR protein expression did not differ, but placental TfR expression was lower in USR fetuses at GD130. Placental levels of TfR correlated to eNOS. TfR was localized throughout the placentome, including the hemophagous zone, implicating a role for TfR in ovine placental iron transport.

CONCLUSION: Fetal iron was regulated in an organ-specific manner. In USR fetuses, NO-mediated placental adaptations may prevent the normal upregulation of placental TfR at GD130.

$\mathbf{U}_{\mathrm{p}}$ terine anomalies and multifetal gestations are two independent and increasingly common clinical factors associated with intrauterine growth restriction (IUGR) $(1,2)$. IUGR ultimately increases the risk of adult-onset diseases, including impaired renal development and hypertension, as described by the Barker hypothesis of developmental origins of adult disease (3). Current practices in assisted reproductive technologies increase both the conception rates and multifetal pregnancies in women with uterine anomalies (4). In human twins, hypertension and impaired renal development are reported in the smaller twin (5), but the impact of multifetal gestation or reduced space for placental development on fetal kidney development remains unknown. Sheep can be used to model multifetal gestation and the impact of reduced space on placental function, fetal growth, and renal development (6,7). An advantage of using the sheep model is that nephrogenesis ends at $80 \%$ of gestation in sheep as reported in humans (8).

An important pathological etiology in IUGR is the depletion of fetal tissue iron (9). Impaired fetal iron delivery is linked to both renal structural anomalies and hypertension $(10,11)$. However, virtually nothing is known about placental iron transfer in multifetal gestation and/or reduced uterine space. In mammals, iron transport to the fetus is accomplished by three recognized pathways: (i) iron-rich endometrial gland secretions, (ii) endocytosis of transferrin (Tf)-bound iron through transferrin receptors (TfRs), and (iii) trophoblast ingestion of pooled maternal erythrocytes in the hemophagous zone (1214). Although detailed mechanisms have not been described in sheep, the ovine model confers the advantage of utilizing all three pathways for fetal iron acquisition (12).

TfR is the major iron transporter in most tissues, including human and rodent placentae $(15,16)$, but its role in sheep has not been described. TfR expression is regulated by cellular iron levels (16). Placental TfR level was increased after mild gestational iron deficiency anemia (IDA) in humans (15) and after IDA-induced IUGR in rats, with the expression inversely related to fetal liver iron levels (16). By contrast, placental TfR expression was lower in singleton human IUGR (17), perhaps because of poor uteroplacental blood flow (9). In addition to iron, nitric oxide (NO), which is produced by the enzyme endothelial nitric oxide synthase (eNOS), can regulate TfR expression (18). Because NO is vital to placental function (19), examining the interplay between placental TfR and NO in the context of multifetal gestation and/or limited uterine space should provide a better understanding of iron transport mechanisms.

We previously reported an ovine uterine space restriction (USR) model (7) with reduced space for placentomal development combined with multifetal gestation that caused asymmetrical IUGR. The role of eNOS and NO in regulating placental TfR expression, fetal iron status, and fetal kidney development in multifetal gestation can be investigated using this model. We hypothesize that placental iron transporter expression in USR will be downregulated by eNOS and reflected by lower 
fetal liver and kidney iron contents. Our aims were to (i) evaluate the impact of limited space for placental development on maternal iron status, placental TfR expression, and fetal liver iron status; (ii) investigate the interplay between iron status and impaired renal development; and (iii) evaluate the association between TfR and eNOS expression in both the placenta and kidney.

\section{RESULTS}

\section{Fetal and Placental Morphometry}

This fetal cohort consisted of 12 non-space restricted (NSR) and 12 USR fetuses at gestational day (GD) 120, and 10 NSR and 19 USR fetuses at GD130 (Table 1). As in our earlier studies (7), this cohort exhibited placental adaptation and lower placental efficiency, greater fetal weight to placental weight ratio, and asymmetric IUGR with brain sparing in USR between GD120 and GD130. Growth arrest in USR at GD130 was seen, as measured by fetal body, kidney, and liver weights (Table 1). Fetal weight $(\mathrm{kg})$ in USR fetuses did not differ from that of NSR fetuses at GD120, but a growth arrest was seen over time, resulting in $40 \%$ lower weight in USR vs. NSR fetuses at GD130 $(P<0.005)$. In USR fetuses, fetal kidney weight (g) was unchanged between GD120 and GD130 but was $19 \%$ lower $(P<0.005)$ and $29 \%$ lower $(P<0.001)$ than those of NSR fetuses at GD120 and GD130, respectively. Mean kidney weight expressed proportionate to fetal weight $(\mathrm{g} / \mathrm{kg})$ in USR did not differ from NSR at either time point, but the ratio $(\mathrm{g} / \mathrm{kg})$ fell $20 \%$ between GD120 and GD130 in both USR and NSR $(P<0.005)$. In USR fetuses, liver weight $(\mathrm{g})$ was unchanged over time but was $17 \%$ lighter $(P<0.05)$ and $33 \%$ lighter $(P<0.0001)$ as compared with NSR at GD120 and GD130, respectively. Liver weight adjusted to fetal weight (g/ $\mathrm{kg}$ ) between USR and NSR fetuses did not differ at either time point, but the ratio in either group was 26\% lower at GD130, as compared with GD120 $(P<0.0001)$ (Table 1$)$.

Compared with NSR, total placentome weight (g) was $56 \%$ heavier in the USR group $(P<0.0001)$ at GD120 and $48 \%$ heavier $(P<0.0001)$ in the USR group at GD130 (Table 1).

Table 1. Fetal morphometrics of non-space restricted and uterine space-restricted fetuses at GD120 and GD130

\begin{tabular}{|c|c|c|c|c|}
\hline & \multicolumn{2}{|c|}{ GD120 } & \multicolumn{2}{|c|}{ GD130 } \\
\hline & Nonrestricted $(n=12)$ & Restricted $(n=12)$ & Nonrestricted $(n=10)$ & Restricted $(n=19)$ \\
\hline Fetal weight (kg) & $2.97 \pm 0.20$ & $2.53 \pm 0.19$ & $4.27 \pm 0.47^{*+}$ & $3.08 \pm 0.17^{\ddagger}$ \\
\hline Kidney weight (g) & $23.0 \pm 1.1$ & $19.5 \pm 1.5$ & $26.5 \pm 2.6^{\dagger}$ & $18.7 \pm 0.7^{*+\neq}$ \\
\hline Kidney weight/fetal weight (g/kg) & $7.9 \pm 0.3$ & $7.8 \pm 0.5$ & $6.3 \pm 0.3^{*+}$ & $6.2 \pm 0.3^{*+}$ \\
\hline Liver weight (g) & $107.4 \pm 4.1$ & $88.9 \pm 6.0^{*}$ & $117.9 \pm 11.5^{\dagger}$ & $78.1 \pm 4.2^{* \neq}$ \\
\hline Liver weight/fetal weight (g/kg) & $37.4 \pm 1.7$ & $35.7 \pm 1.3$ & $28.5 \pm 1.8^{* \dagger}$ & $25.8 \pm 1.3^{* \dagger}$ \\
\hline Placentome weight (g) & $543.5 \pm 24.6$ & $1,223.0 \pm 36.5^{*}$ & $520.5 \pm 52.5^{\dagger}$ & $1,010.0 \pm 31.7^{*+\neq}$ \\
\hline Placentome weight/fetus (g) & $543.5 \pm 24.6$ & $407.5 \pm 12.2^{*}$ & $520.5 \pm 52.5^{\dagger}$ & $318.8 \pm 13.3^{* \dagger \neq}$ \\
\hline
\end{tabular}

Data are means $\pm S E M ; n$, number of fetuses. Superscripts compare non-space restricted and uterine space-restricted values within each gestational day (GD) and between GDs. *Significant difference $(P<0.05)$ as compared with non-space restricted group at GD120; ${ }^{\dagger}$ significant difference $(P<0.05)$ as compared with uterine space-restricted group at GD120; *significant difference $(P<0.05)$ as compared with non-space restricted group at GD130.

Table 2. Erythrocyte iron indexes in maternal artery and fetal vein of non-space restricted and uterine space-restricted groups at GD120 and GD130

\begin{tabular}{|c|c|c|c|c|c|c|c|c|}
\hline & \multicolumn{4}{|c|}{ Maternal } & \multicolumn{4}{|c|}{ Fetal } \\
\hline & \multicolumn{2}{|c|}{ GD120 } & \multicolumn{2}{|c|}{ GD130 } & \multicolumn{2}{|c|}{ GD120 } & \multicolumn{2}{|c|}{ GD130 } \\
\hline & $\begin{array}{l}\text { Nonrestricted } \\
\quad(n=12)\end{array}$ & $\begin{array}{l}\text { Restricted } \\
\quad(n=4)\end{array}$ & $\begin{array}{l}\text { Nonrestricted } \\
\quad(n=10)\end{array}$ & $\begin{array}{l}\text { Restricted } \\
\qquad(n=6)\end{array}$ & $\begin{array}{l}\text { Nonrestricted } \\
\quad(n=12)\end{array}$ & $\begin{array}{l}\text { Restricted } \\
\quad(n=12)\end{array}$ & $\begin{array}{l}\text { Nonrestricted } \\
\quad(n=10)\end{array}$ & $\begin{array}{l}\text { Restricted } \\
\quad(n=19)\end{array}$ \\
\hline Total iron (mg) & $15.9 \pm 2.1$ & $13.2 \pm 0.6$ & $17.1 \pm 1.4^{+}$ & $14.5 \pm 0.4$ & $17.3 \pm 1.4$ & $18.0 \pm 0.8$ & $18.1 \pm 1.1$ & $18.7 \pm 0.6$ \\
\hline $\mathrm{RBCs}$ (million $/ \mathrm{mm}^{3}$ ) & $9.1 \pm 0.9$ & $7.7 \pm 0.2$ & $10.0 \pm 0.7^{\dagger}$ & $8.5 \pm 0.2^{\ddagger}$ & $8.3 \pm 0.7$ & $8.2 \pm 0.4$ & $8.5 \pm 0.5$ & $9.2 \pm 0.3$ \\
\hline RDW (\%) & $26.3 \pm 1.8$ & $25.7 \pm 0.3$ & $27.1 \pm 1.0$ & $25.4 \pm 0.4$ & $23.1 \pm 0.9$ & $24.2 \pm 1.7$ & $23.4 \pm 0.4$ & $25.0 \pm 0.6$ \\
\hline Plasma iron $(\mu \mathrm{g} / \mathrm{dl})$ & $145.4 \pm 9.3$ & $140.0 \pm 6.8$ & $147.8 \pm 9.1$ & $162.6 \pm 10.0$ & $210.1 \pm 13.4$ & $239.7 \pm 8.5$ & $226.6 \pm 19.2$ & $275.9 \pm 11.2^{*+\neq}$ \\
\hline$[\mathrm{Tf}](\mathrm{mg} / \mathrm{l})$ & $354.6 \pm 35.8$ & $300.4 \pm 7.2$ & $317.0 \pm 13.4$ & $326.1 \pm 4.4$ & $274.9 \pm 40.2$ & $233.1 \pm 18.0$ & $311.4 \pm 53.8$ & $511.2 \pm 33.2^{*+\ddagger}$ \\
\hline Tf saturation (\%) & $29.6 \pm 3.0$ & $34.8 \pm 1.2^{*}$ & $36.0 \pm 2.4^{*}$ & $43.9 \pm 1.1^{\text {*tf }}$ & $62.4 \pm 7.2$ & $71.9 \pm 4.1$ & $55.1 \pm 9.0$ & $41.2 \pm 1.5^{*+}$ \\
\hline
\end{tabular}

Data are means \pm SEM; $n$, number of sheep or fetuses. Maternal blood data were compared with maternal blood data. Fetal blood data were compared with fetal blood data. Superscripts compare non-space restricted and uterine space-restricted values within each gestational day (GD) and between GDs.

MCV, mean cell volume; RBC, red blood cell; RDW, red blood cell distribution width; Tf, transferrin; TIBC, total iron-binding capacity. *Significant difference ( $P<0.05)$ as compared with non-space restricted group at GD120; ${ }^{\dagger}$ significant difference $(P<0.05)$ as compared with uterin space-restricted group at GD120; ${ }^{*}$ significant difference $(P<0.05)$ as compared with non-space restricted group at GD130. 
Table 3. Fetal liver nonheme iron in non-space restricted and uterine space-restricted groups at GD120 and GD130

\begin{tabular}{lccccc}
\hline & \multicolumn{2}{c}{ GD120 } & & GD130 \\
\cline { 2 - 3 } & Nonrestricted $(n=6)$ & Restricted $(n=6)$ & & Nonrestricted $(n=7)$ & Restricted $(n=7)$ \\
\hline$\mu \mathrm{g} / \mathrm{g}$ Wet weight & $7.18 \pm 1.04$ & $6.18 \pm 0.95$ & & $8.36 \pm 0.36^{\dagger}$ & $8.36 \pm 1.00$ \\
Total $\mu \mathrm{g}$ & $780.0 \pm 153.6$ & $656.8 \pm 110.9$ & & $1,029.0 \pm 158.1^{\dagger}$ & $655.3 \pm 136.0^{\ddagger}$ \\
$\mu \mathrm{g} / \mathrm{kg}$ Proportional to fetal weight & $268.8 \pm 43.6$ & $222.7 \pm 36.3$ & & $235.7 \pm 17.3$ & $220.7 \pm 30.0$ \\
\hline
\end{tabular}

Data are means \pm SEM; $n$, number of fetuses. Superscripts compare non-space restricted and uterine space-restricted values within each gestational day (GD) and between GDs.

tSignificant difference $(P<0.05)$ as compared with uterine space-restricted group at GD120; ${ }^{\ddagger}$ significant difference $(P<0.05)$ as compared with non-space restricted group at GD130.

Table 4. Fetal kidney nonheme iron in non-space restricted and uterine space-restricted groups at GD120 and GD130

\begin{tabular}{|c|c|c|c|c|}
\hline & \multicolumn{2}{|c|}{ GD120 } & \multicolumn{2}{|c|}{ GD130 } \\
\hline & Nonrestricted $(n=7)$ & Restricted $(n=6)$ & Nonrestricted $(n=6)$ & Restricted $(n=8)$ \\
\hline$\mu \mathrm{g} / \mathrm{g}$ Wet weight & $0.47 \pm 0.06$ & $0.60 \pm 0.06$ & $0.40 \pm 0.01^{\dagger}$ & $0.63 \pm 0.05^{\ddagger}$ \\
\hline$\mu \mathrm{g} / \mathrm{kg}$ Proportional to fetal weight & $3.8 \pm 0.4$ & $5.2 \pm 0.7^{*}$ & $2.4 \pm 0.2^{*+}$ & $3.8 \pm 0.3^{\dagger \neq}$ \\
\hline
\end{tabular}

Placentome weight per individual fetus, i.e., proportionate to fetal number, was $25 \%$ lower $(P<0.001)$ in the USR group at GD120 and 39\% lower $(P<0.001)$ at GD130 as compared with NSR (Table 1).

\section{Maternal and Fetal Circulating Iron Indexes}

Because $75 \%$ of body iron is present in erythrocytes, maternal and fetal red blood cell (RBC) count, hematocrit, and mean cell volume (MCV) were examined (Table 2). No differences were seen at GD120 between groups for any erythrocyte iron index. At GD130, maternal RBC count was 15\% lower $(P<$ 0.05) in USR than NSR, but maternal MCV, plasma iron, and total iron-binding capacity (TIBC) did not differ; however, Tf saturation was $26 \%$ higher $(P<0.05)$. We found no relationship between maternal total mg of circulating iron and total $\mathrm{mg}$ of circulating iron in the combined fetoplacental unit $(R$ $=-0.175 ; P=0.3)$. Fetal RBC counts and calculated total $\mathrm{mg}$ of circulating iron per fetus did not differ between USR and NSR groups at GD130. In USR at GD130, fetal MCV was 6\% lower and fetal Tf saturation was 30\% lower than in NSR $(P<$ $0.05)$. However, because fetal TIBC was $40 \%$ higher $(P<0.05)$, plasma iron was unchanged as compared with NSR (Table 2).

\section{Fetal Liver Iron Assessment}

Fetal liver iron concentration ( $\mu \mathrm{g} / \mathrm{g}$ wet weight) of NSR vs. USR was not different at either GD120 or GD130 (Table 3). At GD120, similar total fetal liver nonheme iron allotment $(\mu \mathrm{g})$ was seen, but at GD130, USR was 36\% lower than NSR $(P<$ $0.05)$. Liver iron expressed proportionate to fetal weight $(\mu \mathrm{g} /$ $\mathrm{kg}$ ) was similar in the two groups at both times (Table 3 ).

\section{Fetal Kidney Iron Assessment}

Fetal kidney nonheme iron concentration ( $\mu \mathrm{g} / \mathrm{g}$ wet weight) was not different between treatments at GD120 but was greater in
USR at GD130 as compared with NSR (Table 4). Surprisingly, fetal kidney iron allotment $(\mu \mathrm{g})$ in USR was higher than that in NSR at GD120, but it was similar in the two groups at GD130. However, kidney iron was 27\% greater at GD120 and 37\% greater at GD130 in USR than in NSR when adjusted proportionate to fetal weight ( $\mu \mathrm{g} / \mathrm{kg})(P<0.05$ for both comparisons) (Table 4$)$.

\section{Fetal Kidney TfR and eNOS Expression}

Immunohistochemistry for all groups and time points showed TfR expression in the glomeruli, maculae densa, and distal and proximal tubules (Figure 1a). Tubular epithelial TfR staining was seen on both the luminal surface and within endocytic vesicles. Initial assessment of TfR expression in the kidney found virtually no differences in tissue localization or intensity between treatments or between GD groups, so immunoblot was performed instead of quantitative morphometric analysis. Enhanced Prussian blue staining showed intracellular iron deposits in phagocytic cells and within the glomeruli and tubular epithelium in kidneys of some USR fetuses (Figure 1b), but none were observed in NSR. In fetal kidney tissue, normalized TfR protein by immunoblot showed no difference between groups at either gestational age (Figure 2). To determine the relationship between $\mathrm{NO}$ and TfR expression, renal eNOS expression by immunoblot was determined. We observed no difference in renal eNOS expression in either group at either time point $(P>0.05)$ (Figure 2). Kidney TfR expression was not related to kidney eNOS expression $(r=0.02 ; P=0.95)$.

\section{Placentome TfR and eNOS Expression}

For all groups at all time points, TfR staining was found in uterine glands and maternal and fetal components of the placentome (Figure 1c,d). Prominent TfR staining was also localized to the chorionic epithelium of the hemophagous region, with apical microvilli encircling maternal erythrocytes via 


\section{Articles | Sun et al.}
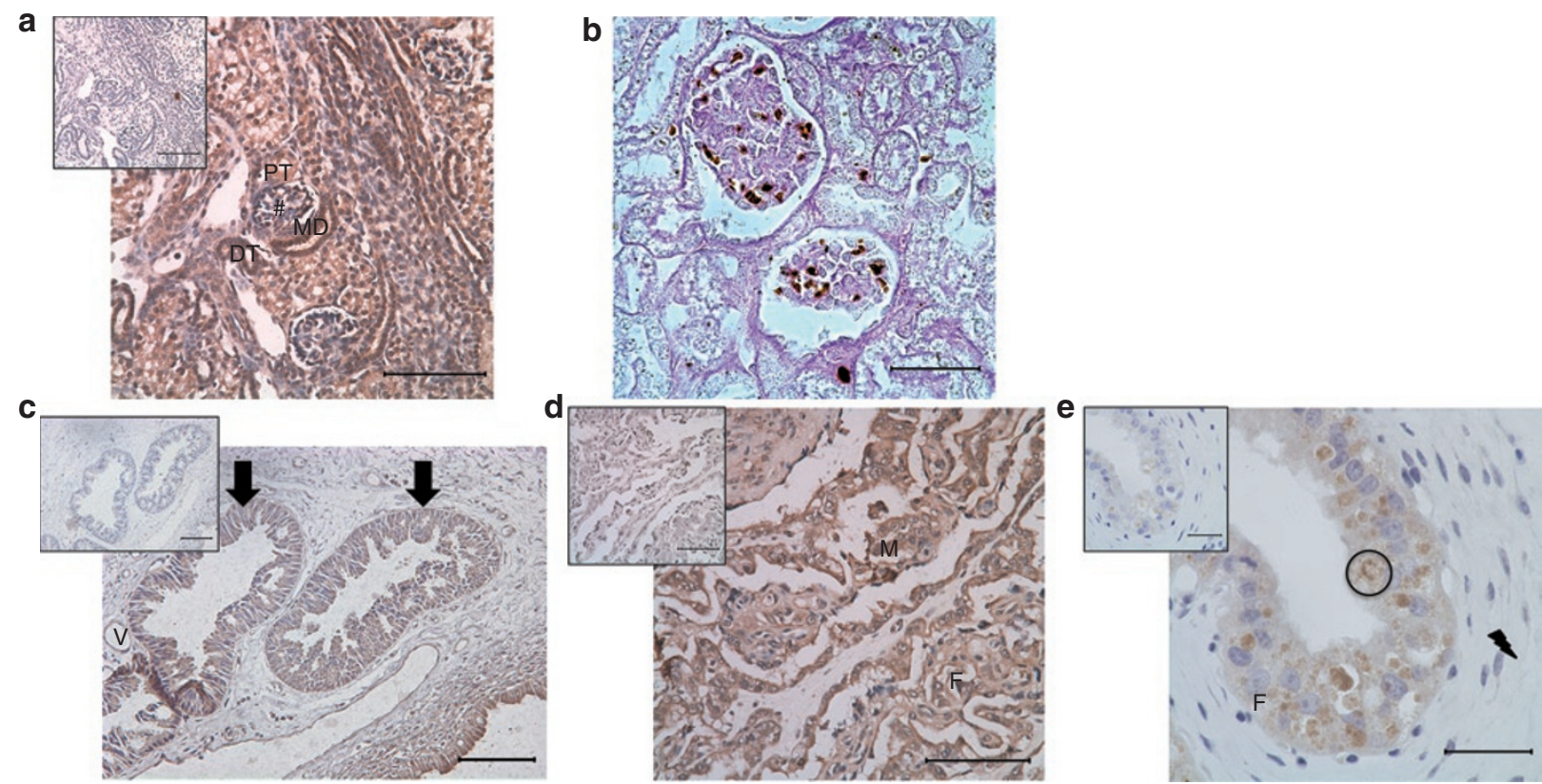

Figure 1. Kidney and placenta histology and immunohistochemistry. (a) DAB brown staining of a NSR at GD120 kidney indicating TfR localization in the distal tubule (DT), maculae densa (MD), proximal tubule (PT), and glomerulus (\#) with immunoglobulin G (IgG)-negative control in the top left corner. Sections were counterstained with hematoxylin. Bar $=100 \mu \mathrm{m}$. (b) Enhanced Prussian blue staining in brown of iron deposits in the glomerulus of a USR at GD130 kidney. Bar $=35 \mu \mathrm{m}$. (c) DAB brown staining of TfR seen primarily in the uterine glands of a NSR at GD130 placenta. Arrows indicate uterine glandular epithelium and interstitial tissue with $(\mathrm{V})$ showing a vessel. Top left corner shows the IgG-negative control. Sections were counterstained with hematoxylin. Bar $=100 \mu \mathrm{m}$. (d) DAB brown staining of TfR in fetal trophoblasts (F) and maternal epithelium (M) at the interdigitation of the villi and septa of a USR at GD130 placenta with the IgG-negative control in the top left corner. Sections were counterstained with hematoxylin. Bar $=100 \mu \mathrm{m}$. (e) DAB brown staining of a NSR at GD130 placenta indicating TfR localization in the hemophagous zone with the lightning bolt showing the stroma, circle indicating ingested erythrocyte, and fetal villus $(F)$, with lgG negative control on the top left corner. Sections were counterstained with hematoxylin. Bar $=35$ $\mu \mathrm{m}$. DAB, diaminobenzidine; GD, gestational day; NSR, non-space restricted; TfR, transferrin receptor; USR, uterine space restriction.

endocytic vesicles for both groups and time points (Figure 1e). Rust-colored staining of partially degraded erythrocyte hemoglobin (see circles) was seen, but additional staining for TfR also surrounded the encircled erythrocytes (Figure 1e). Initial assessment of placentomal TfR expression found virtually no differences in tissue localization or intensity between treatments or between GD groups, so immunoblot was performed instead of quantitative morphometric analysis. Immunoblot analyses revealed that placental TfR expression was not different between NSR and USR groups at GD120 (Figure 3). However, at GD130, TfR expression was $40 \%$ lower in USR as compared with that in NSR $(P<0.0001)$. In contrast to TfR, as compared with GD120 levels, placental eNOS expression was fourfold higher in NSR $(P<0.005)$ and sixfold higher in USR $(P<0.05)$ by GD130 (Figure 3$)$.

Using all samples regardless of GD and uterine space condition, placental TfR expression was positively correlated with placental eNOS expression $(r=0.726 ; P<0.001)$ (Figure 4), whereas placental TfR and fetal liver nonheme iron did not correlate $(r=0.06 ; P=0.8)$.

\section{DISCUSSION}

This study is the first in-depth examination of the effects of maternal and fetal iron metabolism in IUGR sheep. We utilized our previously described model of IUGR induced by multifetal gestation and restricted surface area for placental development (7) to investigate whether IUGR caused by USR exhibited iron metabolism similar to placental insufficiency-induced IUGR or gestational IDA-induced IUGR. Our findings are important because of the space limitations seen in $5.5 \%$ of human pregnancies with uterine structural anomalies (20) and the $3 \%$ of human pregnancies carrying multiple fetuses (1). Similar to both placental insufficiency and gestational IDA, we observed asymmetric IUGR in USR, with proportionately smaller liver and kidney weights. In USR, we found normal maternal iron status and observed neither decreased nor increased fetal RBC mass. In USR, fetal liver iron $(\mu \mathrm{g})$ was proportionately lower, but kidney iron $(\mu \mathrm{g})$ was similar but higher proportionate to fetal weight as compared with NSR and inconsistent with IUGR from either gestational IDA or placental insufficiency. We are the first to show that ovine placenta uses TfR. Unlike gestational IDA, placental TfR expression was correlated with placental eNOS expression but not liver iron.

The risk for maternal IDA increases fourfold during singleton human pregnancies (21). IDA during pregnancy can contribute to IUGR (21), and carrying multiple fetuses further increases maternal risk for IDA because of greater fetal iron needs (22). Blood hematological indexes were unlike either gestational IDA-induced or placental insufficiency-induced IUGR. Although the USR ewes exhibited lower hemoglobin, 
a

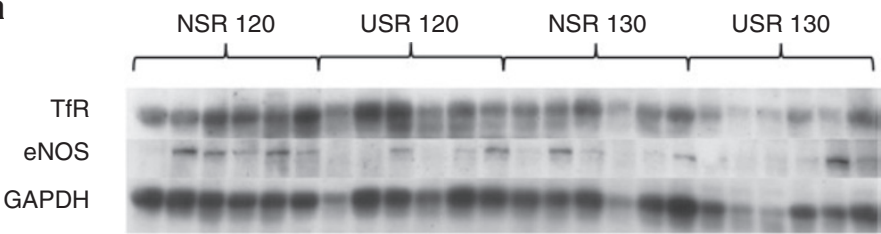

b

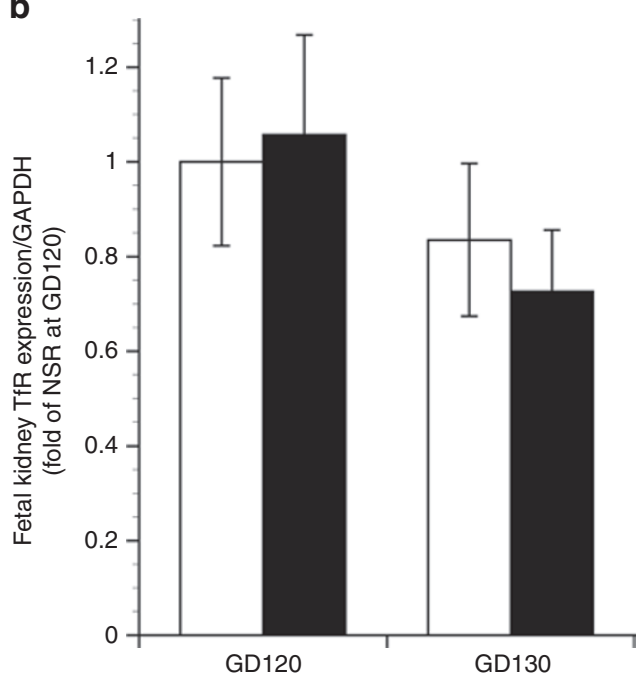

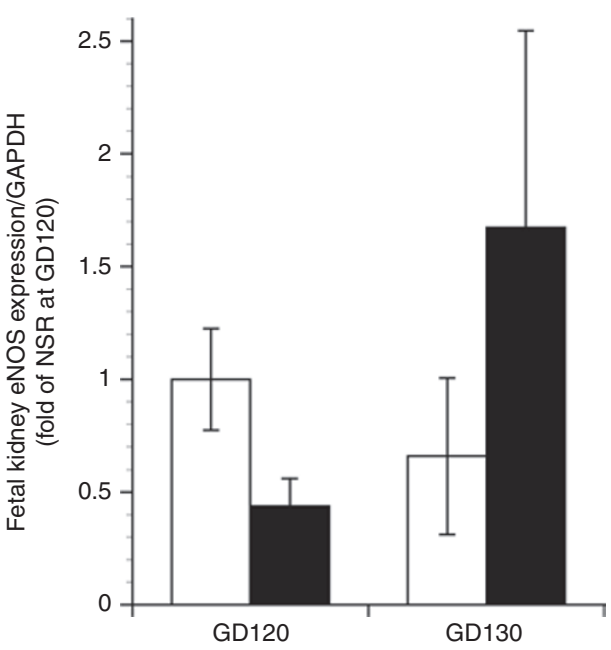

Figure 2. Fetal kidney TfR and eNOS expression in non-space restricted (NSR) and uterine space-restricted (USR) groups at GD120 and GD130. (a) Western blot of TfR and eNOS with GAPDH as loading control in fetal kidney. (b,c) Respective optical density means (fold of control NSR kidney at GD120) and SEM. White bars indicate NSR and black bars indicate USR. eNOS, endothelial nitric oxide synthase; GAPDH, glyceraldehyde 3-phosphate dehydrogenase; GD, gestational day; TfR, transferrin receptor.

a
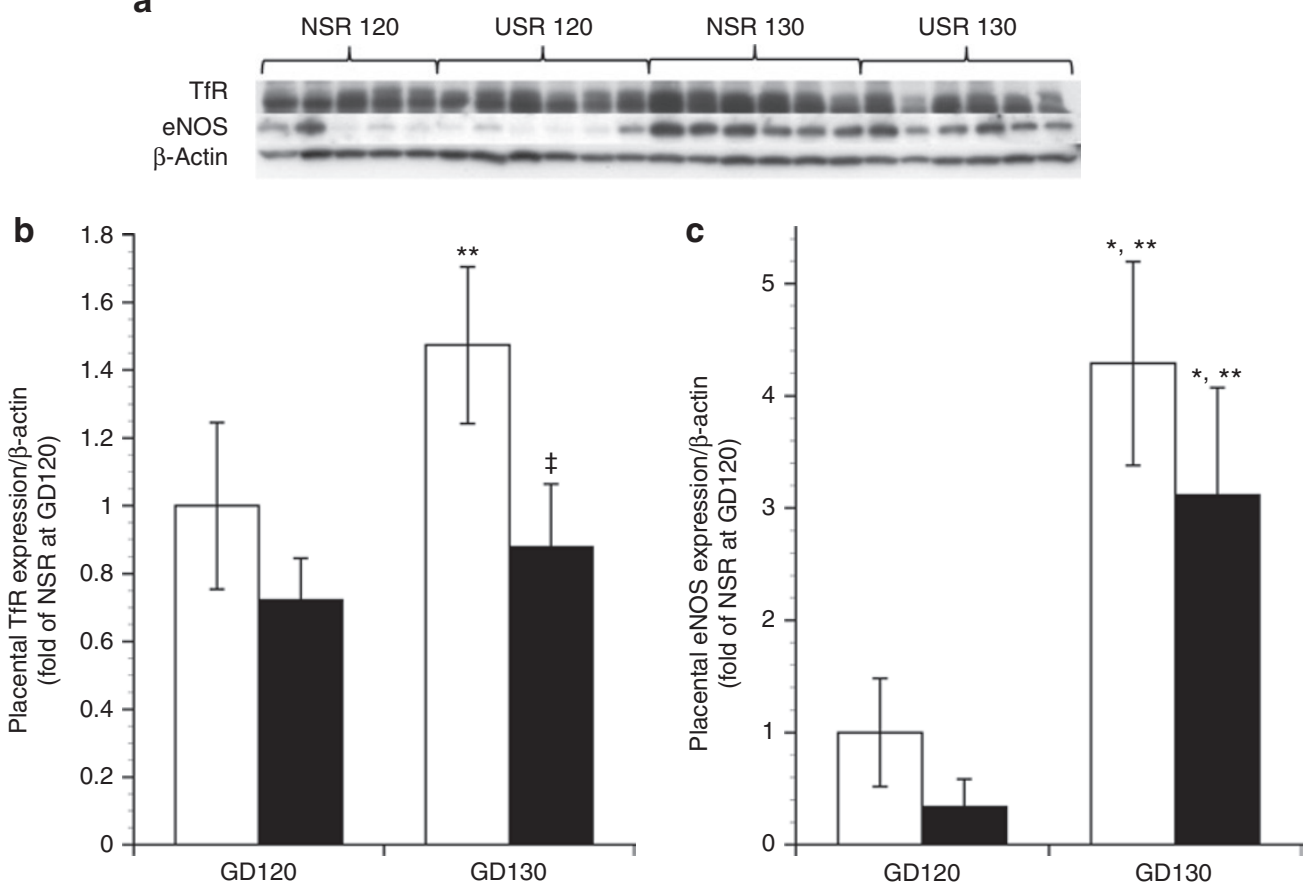

Figure 3. Placental TfR and eNOS expression in non-space restricted (NSR) and uterine space-restricted (USR) groups at GD120 and GD130. (a) Western blot of TfR and eNOS with $\beta$-actin as loading control in the placenta. (b,c) Respective optical density means (fold of control NSR at GD120) and SEM. White bars indicate NSR and black bars indicate USR. *Significant difference $(P<0.05)$ as compared with NSR at GD120; ** significant difference $(P<0.05)$ as compared with USR at GD120; ${ }^{\prime}$ significant difference $(P<0.05)$ as compared with NSR at GD130. eNOS, endothelial nitric oxide synthase; GD, gestational day; TfR, transferrin receptor. 


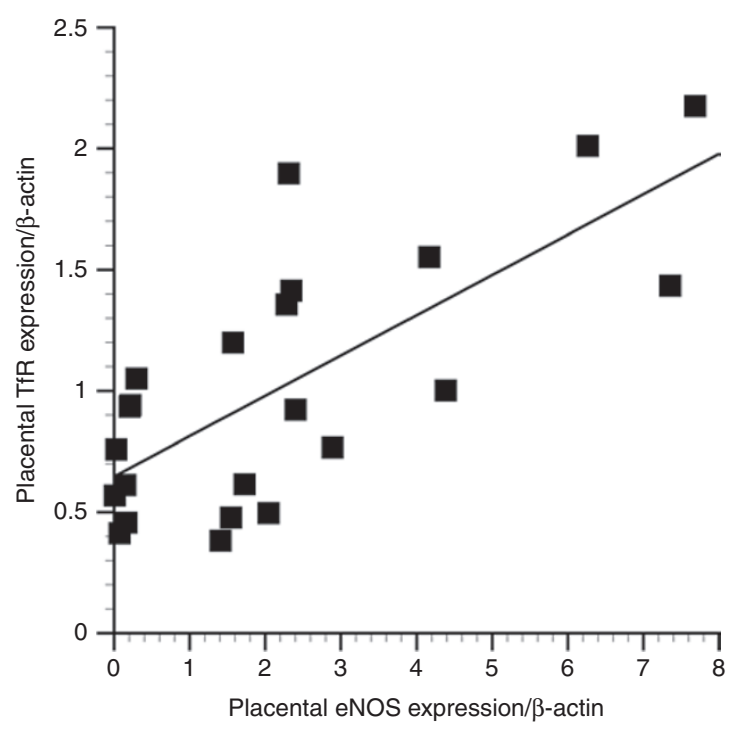

Figure 4. Regulation of placental TfR expression. Placental eNOS expression was quantified and plotted against placental TfR expression $(r=0.726 ; P<0.001)$. eNOS, endothelial nitric oxide synthase; TfR, transferrin receptor.

higher maternal Tf saturation supported normal ewe iron status. The fetuses were not anemic but were mildly microcytic (smaller MCV). Fetal MCV is a suboptimal index of deficient iron status because immature RBCs are larger (higher MCV) than mature RBCs (23). Although fetuses exhibit a greater proportion of immature RBCs, it is likely that smaller fetal MCV represents relatively fewer immature fetal RBCs produced resulting from the growth arrest. Although we found greater fetal TIBC (via higher Tf levels), circulating plasma iron was higher in USR at GD130, inconsistent with fetal iron depletion. On the basis of the calculated total iron needs of the combined fetoplacental units, we found no association between maternal supply and fetal demand. These data support the notion that the USR ewe adapted to the greater fetal iron body mass (and iron needs) by increasing ewe plasma Tf saturation. The fetuses adapted to USR by increasing plasma transport iron capacity. These findings are distinct from human or animal gestational IDA-induced IUGR $(16,24)$. In addition, unlike placental insufficiency-induced IUGR, the USR group had normal ewe hematocrit, ewe iron status (25), and fetal liver iron concentration (26).

Fetal renal iron depletion is reported to accompany IUGR and impair renal development (10). The current study is the first to directly measure fetal renal iron levels after any etiologic cause of IUGR. We observed that liver iron was proportionate to fetal weight, but kidney iron was conserved and regulated on the basis of the amount needed to support normal growth. In contrast to our hypothesis of depleted kidney iron in USRinduced IUGR, the reverse was seen. The apparent difference in how kidney iron is regulated vs. liver iron could be due to the umbilical vein anatomy delivering iron directly to the liver before other fetal organs. The liver is critical for iron storage and is exquisitely sensitive to iron overload, necessitating tight iron regulatory mechanisms via hepcidin (27). Liver hepcidin serves as the master regulator of iron homeostasis, controlling liver iron uptake or release from hepatocytes via plasma Tf. In gestational IDA, hepcidin plays a major role in controlling fetal liver iron (16). However, unlike liver, hepcidin does not control kidney iron trafficking (28), providing a potential explanation for the differences in organ iron regulation.

Fetal renal tubular cells possess luminal TfR and import Tf-bound iron from the filtered urine, instead of importing via plasma Tf, which possibly explains why there was no observed association between renal eNOS and TfR (28). Renal iron content $(\mu \mathrm{g})$ could be determined by the relatively constant fetal glomerular filtration. We also observed TfR staining of tubules, including the specialized distal tubules called the maculae densa, key regulators of renin release from the juxtaglomerular cells. The renin-angiotensin system plays a key role in nephrogenesis (29), in addition to its role in regulating salt balance and blood pressure. The interplay between renin-angiotensin signaling and renal iron concentration has been described (29) but not previously in fetuses. Thus, the greater renal iron could be an adaptive protective mechanism for current survival needs, especially in light of needs for ongoing nephrogenesis and tubular elongation. However, consistent with the developmental origins of adult disease, this response could also be maladaptive in the long term by altering the expression of key renal genes (3). On the basis of higher renal iron content, TfR expression should have been downregulated, but it was not. Furthermore, iron 2+/3+ deposits on enhanced Prussian blue staining are not normally seen in kidneys but were seen in tubules, glomeruli, and phagocytic cells in some USR fetuses. The combination of high iron concentrations and iron deposits suggests that iron excess, instead of deficiency, may disrupt nephrogenesis due to the catalytic potential of unbound iron, which warrants further investigation.

Fetal liver was examined because it regulates plasma iron and Tf levels and storage iron, and also controls placental iron transport, on the basis of fetal iron needs $(16,26)$. In both the USR and NSR groups, fetal liver iron appears to be regulated proportionate to fetal weight. In contrast to the lower blood and tissue iron seen in term human fetuses with placental insufficiency-induced IUGR (26), the current study observed normal fetal blood iron content but greater iron-binding capacity, effectively delivering a proportionate amount of iron to the fetal liver. The difference between the current findings and other models of IUGR could be timing, as we studied fetuses at $80-90 \%$ of term gestation and not those at term. The current data also directly contrast to the findings of lower fetal liver iron correlating to placental TfR in the rat gestational IDA model (16). These conflicting results may be due to supply-demand relationships, with our study demonstrating greater ewe iron supply permitting placental adaptation (7). However, the current study is partly consistent with rat studies that found no relationship between placental TfR and fetal liver iron until liver iron fell below a critical level (16).

TfR, the major iron transporter in the mammalian placenta (16), was 40\% lower in USR than NSR at GD130. This finding was less striking than threefold lower TfR protein expression 
in IUGR placentae of human placental insufficiency singletons (17) or the threefold higher placental TfR mRNA expression in rats with gestational IDA (16). There appears to be no developmental difference in pattern of distribution of TfR between GD120 and GD130, perhaps because the hemophagous zone is fully developed and both time points are temporally located during the rapid growth trajectory that drives iron transfer. A greater difference in distribution might be seen with sampling in early second trimester or term (GD147).

TfR levels are regulated by both intracellular iron and NO $(30,31)$. NO is a rapidly acting molecule catalyzed by NO synthase with high affinity for iron-containing metalloproteins (31). Ovine placental eNOS levels normally rise during gestation, promoting uterine artery dilation via NO (19). Placental eNOS correlates to NO levels, with lower expression in sheep or human IUGR (32). In vivo and in vitro studies (18) show that NO may increase levels from greater TfR mRNA stability (33). Stable placental TfR expression is seen in normal human pregnancy (24), lower expression in placental insufficiency (17), and higher expression in gestational IDA-induced IUGR (16).

The relationship between NO and TfR in placenta was studied. Consistent with previous work (19), placental eNOS levels increased as gestation progressed in both USR and NSR but trended lower in USR than NSR at both time points. However, kidney eNOS expression is reported to be constant throughout gestation and between treatments (34). NO, produced by renal NO synthase enzymes, is a major vasodilatory factor in renal and systemic circulations, controlling renal blood pressure (35). We observed no relationship between TfR and eNOS expression in the kidney but found a direct relationship between placental TfR and eNOS, supporting the notion that eNOS plays a role in placental adaptations enabling maternalto-fetal iron transfer. In keeping with this assertion, we previously observed a dramatic rise in uterine blood flow and eNOS in the gravid vs. nongravid horn in pregnancies after unilateral ligation (36). Greater eNOS and NO production improve uterine blood flow (37). Our findings support possible links among NO, blood flow, and maternal-to-fetal iron transport.

The interaction between NO and TfR had not previously been studied in the placenta; thus, our finding of a direct relationship between placental TfR and eNOS is novel and important. Although iron regulates TfR expression via cytosolic iron regulatory proteins, NO also regulates TfR (31). Iron regulatory proteins bind to hairpin structures called iron response elements on untranslated regions of TfR mRNA, on the basis of intracellular levels of either iron or NO (33). NO increases binding of iron regulatory protein-1, improving iron transport (TfR synthesis) and repressing iron storage (33), but the reverse may occur under oxidizing conditions (30). The direct relationship between eNOS and TfR supports a role for NO in upregulating placental TfR synthesis (18).

The ovine model confers the advantage of utilizing all three mechanisms for fetal iron acquisition: through iron-rich endometrial gland secretions, endocytosis of Tf-bound iron from maternal circulation through TfR, and ingestion of maternal erythrocytes $(12,13)$. We report, for the first time, TfR expression in uterine glands, uniform TfR localization on both the maternal villus and the fetal chorionic epithelium, and, most important, the presence of TfR in the hemophagous zone. Hemophagous zones are the sites where maternal erythrocytes pool and internalize via endocytosis into the chorionic epithelium via the microvilli, where erythrocytic hemoglobin is degraded (12), and iron is reported to be transferred to the fetus. However, the complete mechanism for iron transport in hemophagous zones is incompletely elucidated. TfR was localized throughout the epithelia of the hemophagous zone, supportive of a role in iron transport because cell-surface TfRs located on microvilli normally undergo endocytosis. Focusing our investigation on the hemophagous zone enables examination of the important final steps of iron trafficking out of trophoblasts into neighboring interstitium and fetal endothelium. Of note, we found virtually no $\mathrm{Fe}^{2+}$ or $\mathrm{Fe}^{3+} /$ hemosiderin by enhanced Prussian blue staining near the hemophagous zone (data not shown). Trophoblasts are reported to be minor depots of hemosiderin iron (38), and iron not stored is exported through one of the iron exporters (15), taken up by interstitial Tf, and, finally, imported to fetal endothelium via TfR (16). We are the first to report the localization of TfR in both fetal villous and endothelial cells adjacent to the hemophagous zones in ovine placentomes, supporting the role of TfR in transporting iron through the hemophagous zone into fetal circulation.

In summary, this ovine USR IUGR model differs from both placental insufficiency-induced IUGR and gestational IDAinduced IUGR, because we observed fetal liver iron concentration proportionate to body weight. USR fetuses also exhibited proportionately higher kidney iron relative to body weight, but it is unclear whether this adaptation is advantageous or contributes to long-term maladaptation in kidney function. Although fetal kidney iron was regulated differently than fetal liver iron, we hypothesize that placental TfR expression in USR fetuses did not rise to the same degree as in the NSR group to avoid oversaturating the fetal liver, an organ exquisitely susceptible to iron toxicity. TfR plays a role in all three recognized pathways for fetal iron acquisition: in iron-rich endometrial glands, in the maternal-fetal interface, and in the hemophagous zone. The strong relationship between placental levels of eNOS and TfR demonstrates a potential role for NO in mediating placental-to-fetal iron transport to maintain fetal iron content during a compromised uterine placental development. A better understanding of placental and fetal iron trafficking is necessary to elucidate the mechanisms in alterations of fetal development in all etiologies of IUGR.

Table 5. Number of ewes and fetuses within treatment groups

\begin{tabular}{llcl}
\hline \multirow{2}{*}{ NSR } & Treatment & Ewes $(n=32)$ & Fetuses $(n=53)$ \\
& Unilateral & 9 & 9 (All singleton) \\
\multirow{2}{*}{ USR } & Nonunilateral & 13 & 13 (All singleton) \\
& Unilateral & 8 & 25 (Two triplets) \\
& 2 & $\begin{array}{l}6 \text { (Seven triplets, one } \\
\text { quadruplet) }\end{array}$ \\
\hline
\end{tabular}

NSR, non-space restricted fetuses; USR, uterine space-restriction model. 


\section{METHODS}

Animals

Animal protocols were approved by the Research Animal Care and Use Committee, University of Wisconsin-Madison. Ewes of mixed Western breeds $(n=32)$ were group housed and fed a mixture of hay and corn silage that met daily feed requirements of pregnant sheep. Surgical and synchronization procedures were described by Meyer et al. (7). Ewes were randomly assigned to one of two treatment groups: unilateral uterine horn ligation to limit uterine space for placental development or nonunilateral ligation (Table 5). Unilaterally ligated ewes underwent complete disconnection of a single uterine horn and intercornual vascular connections at least 2 mo before breeding to a fertile ram. A synchronization protocol was performed using an intravaginal controlled internal drug release (Progesterone CIDR; $0.3 \mathrm{~g}$; Pfizer, Aukland, New Zealand) for 10-14 d, followed by intramuscular prostaglandin $\mathrm{F}_{2}$ a $(15 \mathrm{mg}$; Pfizer) and equine chorionic gonadotropin (500 IU; EMD Biosciences, San Diego, CA) injections (7). Pregnancy was confirmed using ultrasound by GD60.

\section{Fetal Measurements and Tissue Collection}

We utilized a smaller cohort of fetuses than previously reported (7), with twins excluded to further demarcate USR fetuses from NSR. Nonsurvival surgery using sodium pentobarbital $(50 \mathrm{mg} / \mathrm{ml})$ was performed on either GD120 (120.4; $n=16$ ewes) or GD130 (129.9; $n=16$ ewes); term = 147 d. Approximately $12 \mathrm{ml}$ of heparinized blood from the maternal jugular vein, and simultaneously from the fetal umbilical vein and artery, were collected before fetal removal and euthanasia. Placentomes, fetal liver, and fetal kidneys were weighed and frozen at $-80^{\circ} \mathrm{C}$. Fresh whole blood was analyzed for indexes of erythrocyte iron (RBC, hemoglobin, MCV) using a pocH-100i laboratory analyzer (Sysmex, Mundelein, IL). Blood was centrifuged for $10 \mathrm{~min}$ at $3,600 \mathrm{~g}$ at $4^{\circ} \mathrm{C}$, and plasma stored at $-80^{\circ} \mathrm{C}$ for plasma Tf and Tf saturation. Total circulating iron was calculated as (weight $\times$ hemoglobin $\times 3.42 \times 1.25) / 1,000$. TIBC was measured by the FerroZine method using an Unbound Iron Binding Capacity assay kit (Thermo Scientific, Middletown, VA). Plasma Tf concentration (mg/dl) was calculated as TIBC/2 (39).

Using placentome number and total placentome weight per fetus, fetuses were grouped as NSR (nonunilateral singleton fetuses $(n=9)$ and unilateral singletons $(n=13))$ or USR (nonunilateral triplets $(n=$ $21)$, nonunilateral quadruplets $(n=4)$, and unilateral triplets $(n=6))(7)$, with treatment and gestation groupings as shown in Table 5. NSR was defined as one fetus per horn, whereas USR was defined as more than one fetus per horn.

\section{Liver and Kidney Nonheme Iron Assay}

Fetal liver and kidneys were homogenized in iron-free water with an ironfree pestle homogenizer. Kidney and liver nonheme iron concentration $(\mu \mathrm{g} / \mathrm{g}$ wet weight), total nonheme iron allotment $(\mu \mathrm{g})$, and total nonheme iron content proportional to fetal weight $(\mu \mathrm{g} / \mathrm{kg})$ were quantified (40).

\section{Kidney and Placentome Histology and Immunohistochemistry}

Formalin-fixed paraffin-embedded fetal kidneys underwent histologi-

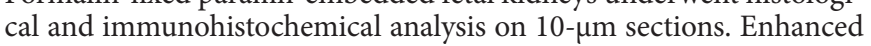
Prussian blue staining was performed to identify iron 2+ and 3+ deposits (41). Immunohistochemistry was used to examine TfR distribution (19). Slides were deparaffinized with HistoClear (National Diagnostics, Atlanta, GA) and rehydrated in graded ethanol. Peroxidase activity was blocked with $3 \%$ hydrogen peroxide. Antigen retrieval step was performed by boiling the tissue in a $0.1 \mathrm{~mol} / \mathrm{l}$ citrate buffer (42). Primary rabbit polyclonal antihuman TfR1 antibody (1:50; Santa Cruz Biotechnology, Santa Cruz, CA), rabbit monoclonal anti-TfR antibody (1:50; Santa Cruz Biotechnology), and biotinylated antirabbit immunoglobulin $\mathrm{G}$ secondary antibody from Vector Labs' Universal ABC Kit (Vector Labs, Burlingame, CA) were used. The tissue was stained with diaminobenzidine (Vector Labs) and counterstained with hematoxylin (Fisher Scientific, Pittsburgh, PA).

\section{Kidney and Placentome Western Blot}

Either frozen placentome or fetal kidney was homogenized in mitogen-activated protein kinase lysis buffer and centrifuged at $16,000 \mathrm{~g}$ for $15 \mathrm{~min}$ at $4^{\circ} \mathrm{C}$. Immunoblot analysis was performed as previously described (43). Monoclonal anti-TfR1 CD71 (1:5,000; Santa Cruz Biotechnology), monoclonal eNOS (1:3,000; BD Laboratories, Franklin Lakes, NJ), monoclonal $\beta$-actin $(1: 3,000$; Cell Signaling, Danvers, MA), and monoclonal glyceraldehyde 3-phosphate dehydrogenase (1:3,000; BD Laboratories) were used. Membranes were visualized with an ECL detection kit (Fisher Scientific). Films were developed and quantified using densitometry.

\section{Data Analysis}

Statistical analyses were performed using a two-way ANOVA followed by a Student-Newman-Keuls post hoc test and simple linear regression using SigmaStat 1.0 software (Jandel Corporation, San Rafael, CA). Immunoblot optical densities were normalized to either $\beta$-actin (placenta) or glyceraldehyde 3-phosphate dehydrogenase (kidney) expression and further standardized as fold difference of NSR at GD120. Data are presented as means \pm SEM, with $P<0.05$ considered significant.

\section{ACKNOWLEDGMENTS}

Manuscript assistance was provided by Sheikh Omar Jobe, Timothy J. Morschauser, and Mayra B. Pastore. Technical support was provided by Jason L. Austin, Kreg M. Grindle, Gladys E. Lopez, Terrance M. Phernetton, and Patrick J. Halbach, as well as Todd A. Taylor from the University of Wisconsin Arlington Farm Facility. The current article has minimal overlap with the previously published data of Meyer et al. (7). This work is in partial fulfillment of the PhD degree of the Interdepartmental Graduate Program in Nutritional Sciences.

\section{STATEMENT OF FINANCIAL SUPPORT}

National Institutes of Health grants HL49210, HD38843, HL087144 (to R.R.M.), and HL087144 Supplement (to P.J.K.); a grant from the Meriter Foundation (to P.J.K.); and a grant from the University of Wisconsin Department of Pediatrics (to P.J.K.) supported the study. The School of Medicine and Public Health funded J.M.H.

Disclosure: The authors declared no conflict of interest.

\section{REFERENCES}

1. Martin JA, Hamilton BE, Sutton PD, Ventura SJ, Mathews TJ, Osterman MJ. Births: final data for 2008. Natl Vital Stat Rep 2010;59(1):1, 3-71.

2. Resnik R. Intrauterine growth restriction. Obstet Gynecol 2002;99:490-6.

3. Barker DJ, Eriksson JG, Forsén T, Osmond C. Fetal origins of adult disease: strength of effects and biological basis. Int J Epidemiol 2002;31:1235-9.

4. Monteagudo A, Strok I, Greenidge S, Timor-Tritsch IE. Quadruplet pregnancy: two sets of twins, each occupying a horn of a septate (complete) uterus. J Ultrasound Med 2004;23:1107-11; quiz 1112-3.

5. Bergvall N, Iliadou A, Johansson S, et al. Genetic and shared environmental factors do not confound the association between birth weight and hypertension: a study among Swedish twins. Circulation 2007;115:2931-8.

6. Gootwine E, Spencer TE, Bazer FW. Litter-size-dependent intrauterine growth restriction in sheep. Animal 2007;1:547-64.

7. Meyer KM, Koch JM, Ramadoss J, Kling PJ, Magness RR. Ovine surgical model of uterine space restriction: interactive effects of uterine anomalies and multifetal gestations on fetal and placental growth. Biol Reprod 2010;83:799-806.

8. Wintour EM, Moritz KM, Johnson K, Ricardo S, Samuel CS, Dodic M. Reduced nephron number in adult sheep, hypertensive as a result of prenatal glucocorticoid treatment. J Physiol (Lond) 2003;549(Pt 3):929-35.

9. Georgieff MK, Mills MM, Gordon K, Wobken JD. Reduced neonatal liver iron concentrations after uteroplacental insufficiency. J Pediatr 1995; 127:308-14.

10. Lisle SJ, Lewis RM, Petry CJ, Ozanne SE, Hales CN, Forhead AJ. Effect of maternal iron restriction during pregnancy on renal morphology in the adult rat offspring. Br J Nutr 2003;90:33-9.

11. Gambling L, Dunford S, Wallace DI, et al. Iron deficiency during pregnancy affects postnatal blood pressure in the rat. J Physiol (Lond) 2003;552(Pt 2):603-10.

12. Burton GJ, Samuel CA, Steven DH. Ultrastructural studies of the placenta of the ewe: phagocytosis of erythrocytes by the chorionic epithelium at the central depression of the cotyledon. Q J Exp Physiol Cogn Med Sci 1976;61:275-86. 
13. Myagkaya GL, Schornagel K, van Veen H, Everts V. Electron microscopic study of the localization of ferric iron in chorionic epithelium of the sheep placenta. Placenta 1984;5:551-8.

14. Pereira FT, Braga FC, Burioli KC, et al. Transplacental transfer of iron in the water buffalo (Bubalus bubalis): uteroferrin and erythrophagocytosis. Reprod Domest Anim 2010;45:907-14.

15. Li YQ, Yan H, Bai B. Change in iron transporter expression in human term placenta with different maternal iron status. Eur J Obstet Gynecol Reprod Biol 2008;140:48-54.

16. Gambling L, Czopek A, Andersen HS, et al. Fetal iron status regulates maternal iron metabolism during pregnancy in the rat. Am J Physiol Regul Integr Comp Physiol 2009;296:R1063-70.

17. Mandò C, Tabano S, Colapietro $P$, et al. Transferrin receptor gene and protein expression and localization in human IUGR and normal term placentas. Placenta 2011;32:44-50.

18. Wang H, Duan X, Liu J, Zhao H, Liu Y, Chang Y. Nitric oxide contributes to the regulation of iron metabolism in skeletal muscle in vivo and in vitro. Mol Cell Biochem 2010;342:87-94.

19. Zheng J, Li Y, Weiss AR, Bird IM, Magness RR. Expression of endothelial and inducible nitric oxide synthases and nitric oxide production in ovine placental and uterine tissues during late pregnancy. Placenta 2000;21:516-24.

20. Chan YY, Jayaprakasan K, Zamora J, Thornton JG, Raine-Fenning N, Coomarasamy A. The prevalence of congenital uterine anomalies in unselected and high-risk populations: a systematic review. Hum Reprod Update 2011;17:761-71.

21. Scholl TO. Iron status during pregnancy: setting the stage for mother and infant. Am J Clin Nutr 2005;81:Suppl:s1218-22.

22. Bdolah Y, Lam C, Rajakumar A, et al. Twin pregnancy and the risk of preeclampsia: bigger placenta or relative ischemia? Am J Obstet Gynecol 2008;198:428.e1-6.

23. Blohowiak SE, Chen ME, Repyak KS, et al. Reticulocyte enrichment of zinc protoporphyrin/heme discriminates impaired iron supply during early development. Pediatr Res 2008;64:63-7.

24. Bradley J, Leibold EA, Harris ZL, et al. Influence of gestational age and fetal iron status on IRP activity and iron transporter protein expression in third-trimester human placenta. Am J Physiol Regul Integr Comp Physiol 2004;287:R894-901.

25. Soubasi V, Petridou S, Sarafidis K, et al. Association of increased maternal ferritin levels with gestational diabetes and intra-uterine growth retardation. Diabetes Metab 2010;36:58-63.

26. Chockalingam UM, Murphy E, Ophoven JC, Weisdorf SA, Georgieff MK. Cord transferrin and ferritin values in newborn infants at risk for prenatal uteroplacental insufficiency and chronic hypoxia. J Pediatr 1987;111:283-6.

27. Pietrangelo A. Hemochromatosis: an endocrine liver disease. Hepatology 2007;46:1291-301.

28. Smith CP, Thévenod F. Iron transport and the kidney. Biochim Biophys Acta 2009;1790:724-30.
29. Ishizaka N, Saito K, Furuta K, et al. Angiotensin II-induced regulation of the expression and localization of iron metabolism-related genes in the rat kidney. Hypertens Res 2007;30:195-202.

30. Kim S, Ponka P. Nitric oxide-mediated modulation of iron regulatory proteins: implication for cellular iron homeostasis. Blood Cells Mol Dis 2002;29:400-10.

31. Mladenka P, Simunek T, Hübl M, Hrdina R. The role of reactive oxygen and nitrogen species in cellular iron metabolism. Free Radic Res 2006;40:263-72.

32. Ziebell BT, Galan HL, Anthony RV, Regnault TR, Parker TA, Arroyo JA. Ontogeny of endothelial nitric oxide synthase mRNA in an ovine model of fetal and placental growth restriction. Am J Obstet Gynecol 2007;197:420.e1-5.

33. Hentze MW, Kühn LC. Molecular control of vertebrate iron metabolism: mRNA-based regulatory circuits operated by iron, nitric oxide, and oxidative stress. Proc Natl Acad Sci USA 1996;93:8175-82.

34. Smith CA, Santymire B, Erdely A, Venkat V, Losonczy G, Baylis C. Renal nitric oxide production in rat pregnancy: role of constitutive nitric oxide synthases. Am J Physiol Renal Physiol 2010;299:F830-6.

35. Mount PF, Power DA. Nitric oxide in the kidney: functions and regulation of synthesis. Acta Physiol (Oxf) 2006;187:433-46.

36. Magness RR, Meyer KM, Sun MY, Ramadoss J, Kling PJ. Development of an ovine surgical model of uterine space restriction: uterine anomalies and multi-fetal gestations on placental growth and uterine vascular adaptations. J Develop Orig Health Dis 2011;2:Suppl 1:S95-6.

37. Magness RR, Sullivan JA, Li Y, Phernetton TM, Bird IM. Endothelial vasodilator production by uterine and systemic arteries. VI. Ovarian and pregnancy effects on eNOS and NO(x). Am J Physiol Heart Circ Physiol 2001;280:H1692-8.

38. Khong TY, Toering TJ, Erwich JJ. Haemosiderosis in the placenta does not appear to be related to chronic placental separation or adverse neonatal outcome. Pathology 2010;42:119-24.

39. Saarinen UM, Siimes MA. Developmental changes in serum iron, total iron-binding capacity, and transferrin saturation in infancy. J Pediatr 1977;91:875-7.

40. Rebouche CJ, Wilcox CL, Widness JA. Microanalysis of non-heme iron in animal tissues. J Biochem Biophys Methods 2004;58:239-51.

41. Schroeter M, Saleh A, Wiedermann D, Hoehn M, Jander S. Histochemical detection of ultrasmall superparamagnetic iron oxide (USPIO) contrast medium uptake in experimental brain ischemia. Magn Reson Med 2004;52:403-6.

42. Shi SR, Chaiwun B, Young L, Cote RJ, Taylor CR. Antigen retrieval technique utilizing citrate buffer or urea solution for immunohistochemical demonstration of androgen receptor in formalin-fixed paraffin sections. J Histochem Cytochem 1993;41:1599-604.

43. JobeSO, Ramadoss J, Koch JM,Jiang Y,Zheng J, Magness RR. Estradiol-17beta and its cytochrome P450- and catechol-O-methyltransferase-derived metabolites stimulate proliferation in uterine artery endothelial cells: role of estrogen receptor-alpha versus estrogen receptor-beta. Hypertension 2010;55: 1005-11. 